Robert Ménard Philippe Sansonetti

\section{ADRESSE}

R. Ménard: docteur en médecine, ancien interne des hôpitaux de Paris, actuellement boursier EMBO, department of pathology, université de New York, Etats-Unis. Ph. Sansonetti : docteur en médecine, professeur à l'Institut Pasteur. Unité de pathogénie microbienne moléculaire, Inserm U. 389, Institut Pasteur, 28, rue du Dr-Roux, 75724 Paris Cedex 15, France.

\section{TIRÉS À PART}

Ph. Sansonetti.

\title{
Signaux moléculaires induisant l'entrée des bactéries entéropathogènes dans les cellules épithéliales: convergences et paradoxes
}

Les bactéries entéropathogènes des genres Shigella, Salmonella et Yersinia ont en commun la capacité de franchir la barrière intestinale. Dans les modèles de cellules épithéliales en culture, elles induisent d'importantes modifications structurales des cellules et peuvent toutes détourner les fonctions du cytosquelette de la cellule au profit de leur entrée. Cependant, malgré d'évidentes analogies phénotypiques dont rendent compte de nombreuses homologies retrouvées parmi les gènes impliqués dans l'invasion des cellules, il existe des différences de stratégie que reflète la diversité physiopathologique des infections causées par ces pathogènes. Pour chacune d'elles est ici présenté l'état des connaissances sur les bases cellulaires et moléculaires de la relation hôte-pathogène, en analysant les signaux cellulaires transduits et leurs effecteurs bactériens.

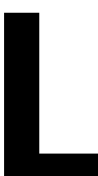

es bactéries des genres Shigella, Salmonella et des espèces entéropathogènes de Yersinia sont responsables de maladies envahissant l'intestin chez l'homme. Elles ont en commun de franchir la barrière intestinale essentiellement via les cellules M [1-5], cellules spécialisées de l'épithélium qui recouvrent les structures lymphoïdes de la muqueuse. En revanche, leur devenir dans la barrière et leur pouvoir pathogène sur les cellules de l'hôte les opposent nettement (figure 1). Shigella, qui provoque un syndrome dysentérique, entraîne des destructions majeures de la mu- queuse colo-rectale. Les cellules épithéliales ou des organes lymphoïdes de la muqueuse sont les cibles électives de ces pathogènes qui ne disséminent qu'exceptionnellement audelà de la muqueuse. A l'inverse, Salmonella typhi et les salmonelles paratyphiques, responsables des fièvres typhoïdes et paratyphoïdes, les nombreux sérotypes de salmonelles responsables chez l'homme de gastroentérites d'origine alimentaire (dont S. typhimurium) et les souches de Yersinia responsables surtout d'adénites mésentériques ( $Y$. pseudotuberculosis et $Y$. enterocolitica) traversent la barrière intestinale au niveau de l'iléon pour 


\section{RÉFÉRENCES}

1. Perdomo JJ, Cavaillon JM, Huerre M, Ohayon H, Gounon P, Sansonetti PJ. Acute inflammation causes epithelial invasion and mucosal destruction in experimental shigellosis. J Exp Med 1994; 180 : 1307-19.

2. Sansonetti P. Stratégie d'infection des cellules épithéliales par les bactéries invasives, étude du modèle Shigella. médecine/sciences 1990; suppl 7: 40-6.

3. Jones BD, Ghori N, Falkow S. Salmonella typhimurium initiates murine infection by penetrating and destroying the specialized epithelial M cells of the Peyer's patches. $J$ Exp Med 1994; 180 : 15-23.

4. Grützkau A, Hanski C, Hahn H, Riecken EO. Involvement of $\mathrm{M}$ cells in the bacterial invasion of Peyer's patches: a common mechanism shared by Yersinia enterocolitica and other enteroinvasive bacteria. Gut 1990 ; $31: 1011-5$

5. Cornélis G. Le plasmide pW, élément clé de la virulence des Yersinia. médecine/sciences 1995; 11 : 1295-304.

6. Clerc P, Sansonetti PJ. Entry of Shigella flexneri into HeLa cells: evidence for directed phagocytosis involving actin polymerization and myosin accumulation. Infect Immun $1987 ; 55: 2681-8$.

7. Adam T, Arpin M, Prévost MC, Gounon $\mathrm{P}$, Sansonetti PJ. Cytoskeletal rearrangements and functional role of T-plastin during entry of Shigella flexneri into HeLa cells. J Cell Biol 1995; 129 : 367-81.

8. Dehio C, Prévost MC, Sansonetti PJ. Invasion of epithelial cells by Shigella flexneri induces tyrosine phosphorylation of cortactin by a pp60 $0^{c-s s c}$ mediated signalling pathway. EMBO J 1995 ; 14: 2471-82.

9. Wu H, Parsons JT. Cortactin, an 80/85-kilodalton pp60 $0^{\text {sc }}$ substrate, is a filamentous actin-binding protein enriched in the cell cortex. J Cell Biol 1993; 120 : 1417-26.

10. Ridley AJ, Hall A. The small GTP-binding protein rho regulates the assembly of focal adhesions and actin stress fibers in response to growth factors. Cell 1992; 70 : 389-

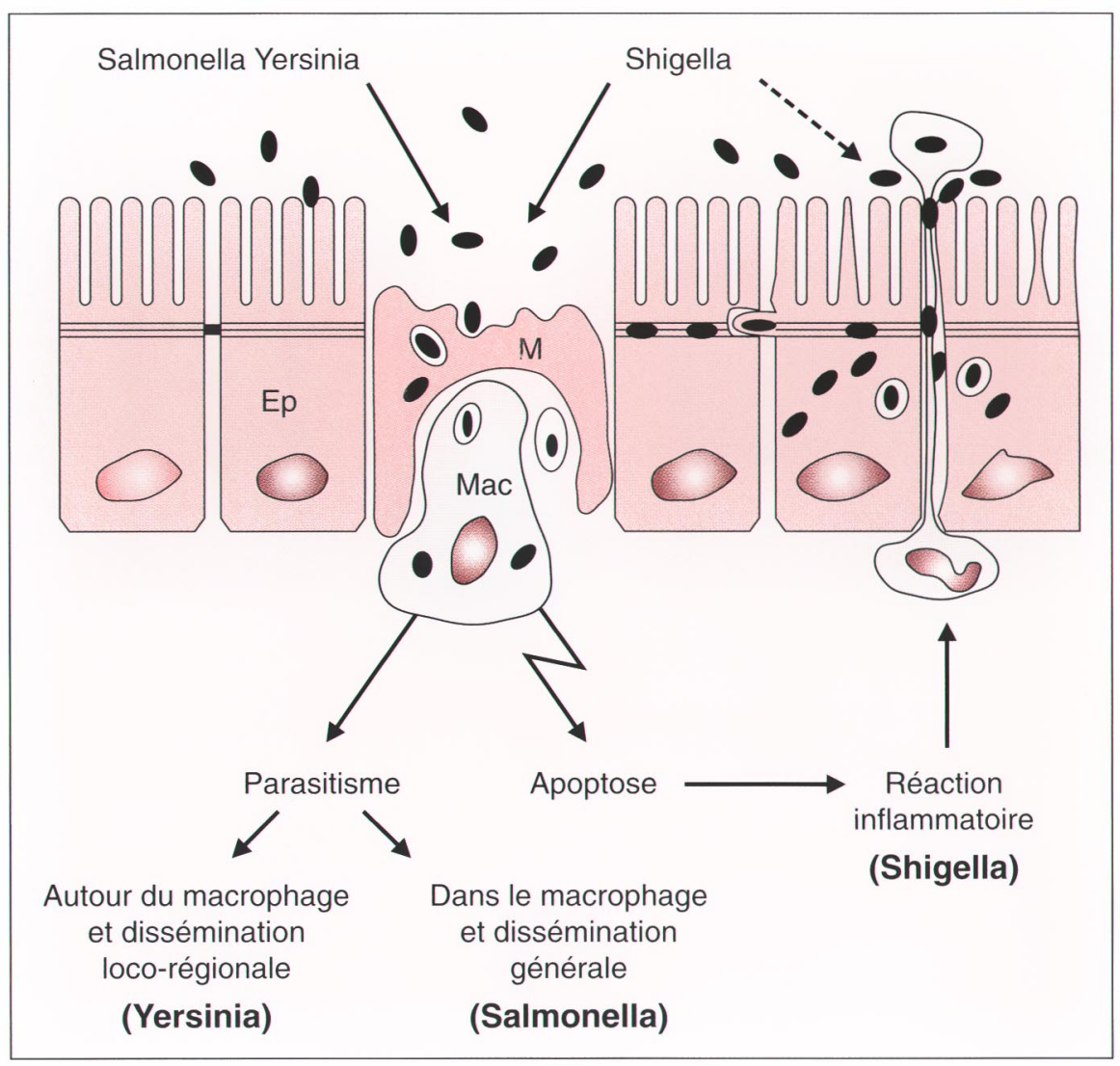

Figure 1. Physiopathologie comparée des infections à Shigella, Yersinia et Salmonella. Shigella, Yersinia et Salmonella pénètrent la barrière intestinale via les cellules $M(M)$, dont le rôle est d'assurer le transfert de particules ou de micro-organismes de la lumière digestive vers les cellules lymphoïdes sous-jacentes. Une partie du pouvoir pathogène des bactéries invasives est de permettre l'attachement spécifique et de faciliter l'entrée dans les cellules $M$ naturellement permissives. Après franchissement de la cellule $M$, l'interaction avec les macrophages (Mac) du follicule lymphö̈de est un véritable carrefour physiopathologique. Une partie essentielle du pouvoir pathogène de Salmonella et de Yersinia, qui envahissent modérément les cellules épithéliales (Ep), est leur potentiel de survie dans ou autour des phagocytes professionnels [50]. A l'inverse, Shigella pénètre dans le macrophage et induit rapidement son apoptose [51], ce qui entraîne le recrutement de polynucléaires $(P N)$ sur le site d'infection. L'intense réaction inflammatoire, et notamment la transmigration de PN entre les entérocytes, permet l'entrée de bactéries luminales par la face basolatérale des cellules et est en grande partie responsable des lésions de la muqueuse [1].

s'établir à distance. Ces bactéries envahissent modérément les cellules épithéliales de la muqueuse intestinale et échappent surtout à l'activité antibactérienne des macrophages associés aux structures folliculaires des sites d'entrée. En adhérant à (Yersinia), ou en survivant dans (Salmonella) les cellules phagocytaires, elles migrent vers les ganglions mésenté- riques et éventuellement les organes à distance.

Toutes ces bactéries entraînent aussi des modifications structurales des cellules épithéliales en culture et peuvent se retrouver en position intracellulaire, dans des vacuoles ou au contact du cytoplasme. Nous exposerons ici les bases moléculaires du pouvoir pathogène de ces bactéries sur les cellules en culture. 


\section{Shigella : une entrée par macropinocytose}

Les cellules HeLa en culture constituent le modèle d'étude in vitro de la virulence de Shigella le plus fréquemment utilisé. Elles permettent de définir et de caractériser les phénotypes majeurs de virulence de la bactérie, comme l'entrée dans la cellule et le mouvement intracellulaire. Cependant, cultivées à confluence, ces cellules épithéliales non intestinales n'établissent pas les relations intercellulaires des cellules différenciées d'un épithélium colique; elles ne permettent donc pas une étude fine de l'interaction entre la bactérie et l'épithélium qui nécessite, outre les modèles animaux d'infection, l'utilisation de systèmes complexes comportant plusieurs populations cellulaires, et notamment des cellules épithéliales polarisées et jointives. Ces systèmes ne seront pas envisagés ici.

Dans les cellules HeLa, l'entrée de Shigella survient surtout au niveau des plaques d'adhérence focale [6], structures dans lesquelles des filaments d'actine et des protéines liant l'actine s'associent à des composants de la matrice extracellulaire par l'intermédiaire des intégrines. Après adhérence, Shigella induit localement d'importantes modifications de la surface cellulaire, comparables à celles induites par les facteurs de croissance ou les agents mitogènes [7]. Des projections cellulaires émergent autour de la bactérie et fusionnent à distance pour englober une ou plusieurs bactéries dans des vacuoles de macropinocytose. Les remaniements de la surface cellulaire sont sous-tendus par des filaments d'actine orientés en faisceaux parallèles et polymérisés à partir de foyers de nucléation d'actine qui apparaissent précocement sous la zone d'interaction bactérie-cellule. La plastine, protéine permettant la compaction de filaments d'actine, est présente dans ces projections et son rôle essentiel dans l'entrée de Shigella a été montré par l'expression transitoire de mutants dominants négatifs pour cette protéine. L'entrée de Shigella s'accompagne aussi de la phosphorylation de résidus tyrosine d'une protéine majoritaire, la cortactine, qui se concentre dans les projections [8].
Cette protéine qui s'associe à l'actine filamenteuse et en particulier aux structures dynamiques du cytosquelette sous-cortical [9], pourrait lier les filaments à la membrane plasmique.

Les petites protéines liant le GTP de type Rho et la protéine $\mathrm{pp} 60^{-s-s c}$ (Src) sont à ce jour les seuls intervenants connus dans la cascade de signalisation aboutissant aux réarrangements du cytosquelette. Les petites GTPases Rho (A, B et C) sont impliquées dans la formation de structures complexes du cytosquelette comme les fibres de tension, les plaques d'adhérence focales ou les projections membranaires [10-12]. Lors de l'entrée de Shigella dans les cellules HeLa, RhoB et RhoC s'accumulent à l'extrémité des projections induites par les bactéries, alors que RhoA s'accumule autour des bactéries en cours d'internalisation [13]. De plus, un inhibiteur spécifique de Rho, la toxine C3 de Clostridium botulinum, empêche la formation des projections induites par Shigella et abolit l'entrée des bactéries. La toxine C3 n'empêche pas l'apparition précoce des points de nucléation d'actine, ce qui suggère que les protéines Rho n'interviennent que secondairement dans l'extension des foyers de nucléation en des projections contenant de longs filaments polymérisés. La protéine Src, membre de la famille des tyrosine kinases membranaires sans domaine récepteur, pourrait être parmi les cibles premières de la bactérie. La protéine Src, qui se concentre dans les plaques d'adhérence et intervient en particulier lors de la croissance et de la différenciation cellulaires [14], est activée et recrutée autour de Shigella en cours d'internalisation et assure la phosphorylation de la cortactine [8]. La surexpression transitoire de Src dans les cellules HeLa entraîne la formation de projections diffuses et provoque l'internalisation de souches non invasives de Shigella. Bien que n'ayant pas encore reçu de démonstration directe, le rôle de Src dans l'entrée de Shigella est donc probable.
19. Ménard R, Sansonetti P, Parsot C. secretion of the Shigella flexneri Ipa invasins is induced by epithelial cells and controlled by IpaB and IpaD. EMBO J 1994; 13: 5293302.

$\mathrm{m} / \mathrm{s} n^{\circ} 4$, vol. 12 , avril 96 


\section{Shigella : une entrée induite par une toxine}

L'étude des bases génétiques de l'entrée de $S$. flexneri dans les cellules a montré qu'une région de $31 \mathrm{~kb}$ d'un grand plasmide retrouvé chez les quatre espèces de Shigella est suffisante pour conférer l'entrée dans les cellules $[15,16]$. Cette région contient plus de trente gènes, dont presque tous ont été étudiés par mutagenèse dirigée [17]. Les protéines codées par cette région et essentielles à l'induction des remaniements du cytosquelette sont les protéines IpgC, IpaB, IpaC, IpaD et les produits d'un appareil de sécrétion, appelé translocon Mxi-Spa, qui permet la sécrétion des trois protéines Ipa dans le milieu de culture (figure 2) [5]. IpgC est un chaperon cytoplasmique qui s'associe à chacune des protéines IpaB et IpaC dans le cytoplasme, assure leur stabilité et empêche leur association [18]. Chez les bactéries cultivées in vitro, l'activité de sécrétion des Ipa est faible et celles-ci s'accumulent dans le cytoplasme bactérien.

Lorsque la bactérie est au contact de la cellule, les protéines Ipa sont massivement libérées dans l'environnement [19]. IpaB et IpaC, mais pas IpaD, s'associent alors dans un complexe soluble qui semble rendre compte de tous les événements cellulaires associés à l'entrée de Shigella [20]. Comme les bactéries invasives, des billes de latex portant ce complexe sont rapidement internalisées dans les cellules en culture au sein de projections membranaires et entraînent des réarrangements du réseau d'actine sous-jacent impliquant les petites protéines Rho. Le mode d'action du complexe d'invasion est encore inconnu. IpaB possède deux régions hydrophobes semblables à certains domaines des $\alpha$-hémolysines et des leucotoxines de la famille RTX, molécules formant des pores dans les membranes de nombreux types de cellules nucléées [21]. IpaB pourrait donc s'insérer dans la membrane de la cellule cible. La seule formation d'un pore, à laquelle pourrait contribuer IpaC, pourrait induire un flux entrant/flux sortant d'ions à l'origine de la cascade de transmission du signal aboutissant à la polymérisation d'actine. L'insertion de
IpaB dans la membrane pourrait, au contraire, permettre la translocation d'un effecteur final, comme IpaC, agissant sur une cible localisée dans le cytosol de la cellule et activant la polymérisation d'actine.

\section{Salmonella, une stratégie d'entrée proche de celle de Shigella}

Comme Shigella, Salmonella est internalisée dans les cellules épithéliales en culture au sein de projections membranaires sous-tendues par une importante filamentation d'actine et une accumulation de protéines liant l'actine [22]. Sur le plan morphologique, rien ne semble distinguer le processus d'entrée de Shigella et de Salmonella. En revanche, les signaux induits par ces deux types de bactéries sont différents.

Les premiers travaux sur la transmission du signal induit par Salmonella ont montré que l'invasion des cellules Henle-407 par S. typhimurium provoquait la phosphorylation de résidus tyrosine d'un certain nombre de protéines, dont le récepteur de l'EGF (epidermal growth factor) [23]. L'activation de ce récepteur aboutit normalement à la stimulation de son activité tyrosine kinase, essentielle aux réponses cellulaires au facteur de croissance, notamment la survenue de projections cellulaires et la réorganisation des microfilaments d'actine [24]. Les voies de signalisation induites par S. typhimurium et l'EGF dans les cellules Henle-407, de même que le profil des protéines phosphorylées semblent identiques : dans les deux cas on observe une augmentation rapide de la concentration de $\mathrm{Ca}^{2+}$ libre intracellulaire $\left(\left[\mathrm{Ca}^{2+}\right]_{\mathrm{i}}\right)$ par flux entrant de $\mathrm{Ca}^{2+}$ extracellulaire [25, 26] (figure 3). Pourtant, le récepteur de l'EGF n'est pas la porte d'entrée exclusive de S. typhimurium, puisque celle-ci peut entrer dans des cellules n'exprimant pas le récepteur de l'EGF [27]. Il semble même que les voies d'activation contrôlées par les petites GTPases Rac (qui contrôlent en particulier le récepteur de l'EGF) ou Rho ne soient pas nécessaires à l'entrée et à la formation des projections induites par Salmonella. Il a donc été suggéré que les effecteurs de l'entrée de Salmonella pourraient reconnaître des déterminants com-
31. Groisman EA, Ochman H. Cognate gene clusters govern invasion of host epithelial cells by Salmonella typhimurium and Shigella flexneri. EMBO J 1993; 12: 3779-87. 


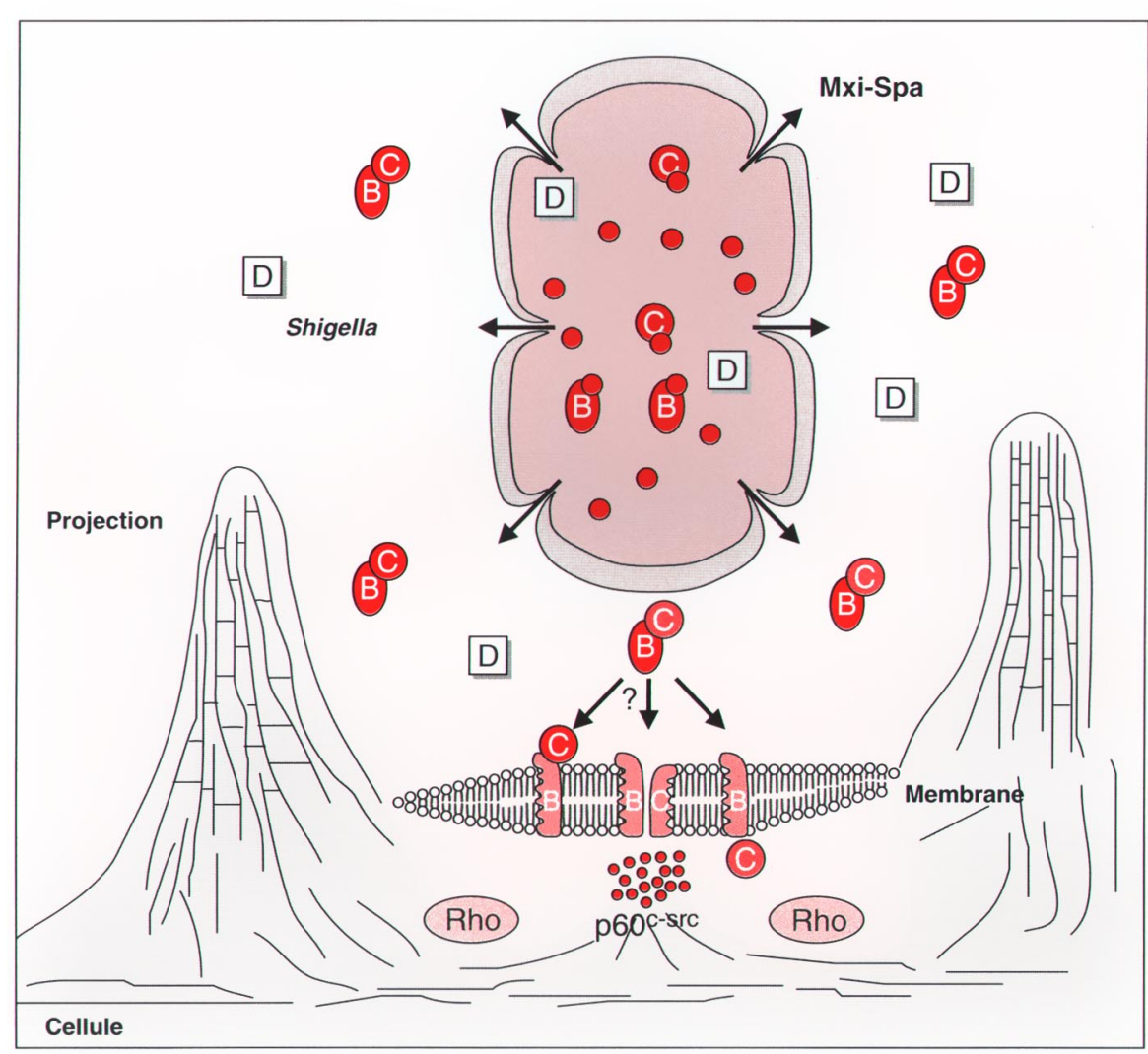

Figure 2. Modèle d'interaction Shigella-cellule. Le système d'entrée de Shigella dans les cellules épithéliales comporte un appareil de sécrétion, le translocon Mxi-Spa, et les quatre protéines $\operatorname{lpg} C(\theta), \operatorname{Ipa} B(B), \operatorname{IpaC}(C)$, et IpaD (D). Shigella pénètre dans la cellule après sécrétion induite par la cellule cible des protéines IpaB et IpaC, qui s'associent en un complexe qui induit la formation de projections membranaires. La libération de IpaB et IpaC est modulée par deux protéines "intermédiaires": IpgC, agissant dans le cytoplasme bactérien pour permettre l'accumulation et la partition des sous-unités du complexe, et IpaD, agissant dans I'appareil de sécrétion spécifique pour assurer leur rétention en l'absence de la cellule cible. Le mode d'action du complexe est inconnu, mais il est probable que IpaB s'insère dans les membranes de la cellule cible pour y former un pore. IpaC pourrait masquer les régions hydrophobes de IpaB avant son interaction avec la membrane, ou s'insérer comme IpaB dans la membrane, ou être transloquée par IpaB et agir sur une cible cytoplasmique. Du côté de la cellule, l'entrée de Shigella induit en particulier le recrutement de petites GTPases de type Rho, nécessaires à l'extension des projections membranaires, et le recrutement sousmembranaire et juxta-bactérien et l'activation de Src. L'hypothèse actuelle est que l'événement initial serait l'induction, au contact de la bactérie, d'une accumulation transmembranaire de pp60 ${ }^{c-s r c}$. Celle-ci pourrait être directement responsable de la formation des projections membranaires, après recrutement et activation d'autres protéines de transmission du signal ou du cytosquelette. muns à une variété de récepteurs, dont le récepteur de l'EGF, qui stimuleraient des voies de signalisation variables avec la lignée cellulaire et le type de récepteur impliqués [28]. Dans les cellules HeLa par exemple, la voie de transmission du signal induit par Salmonella est différente de celle observée dans les cellules Henle-407. Salmonella y provoque une augmentation de la concentration d'inositol triphosphate, probablement par activation de la phospholipase $\mathrm{C} \gamma$, toutes deux nécessaires à l'internalisation des bactéries ainsi qu'à l'augmentation de la $\left[\mathrm{Ca}^{2+}\right]_{\mathrm{i}}$ par libération de stocks intracellulaires de $\mathrm{Ca}^{2+}$ [29].

Quelles que soient les lignées cellulaires utilisées, l'augmentation locale de la $\left[\mathrm{Ca}^{2+}\right]_{\mathrm{i}}$, connue pour régler les fonctions de diverses protéines associées au cytosquelette et entraîner une dépolymérisation rapide d'actine, semble être nécessaire à la formation des projections cellulaires par Salmonella. A l'inverse, aucune variation de la $\left[\mathrm{Ca}^{2+}\right]_{\mathrm{i}}$ n'a été observée après l'entrée de Shigella dans les cellules HeLa [30].

La majorité des travaux génétiques sur l'entrée de Salmonella dans les cellules ont concerné $S$. typhimurium. Tous les gènes d'entrée de cette bactérie décrits à ce jour couvrent une région chromosomique d'environ $40 \mathrm{~kb}$ [4]. La plupart d'entre eux ont un homologue dans la région d'entrée du grand plasmide de Shigella. Notamment, les gènes nommés inv et spa sont analogues des gènes $m x i$ et spa de $S$. flexneri et ont la même organisation [31]; ils permettent aussi la sécrétion des protéines effectrices de l'entrée. Quatre gènes appelés sip, récemment identifiés chez S. typhi et S. typhimurium, sont analogues des gènes ipgC-ipaB-C-D de Shigella et ont une organisation identique [32]. L'ensemble des gènes nécessaires, et probablement suffisants, à l'entrée de Shigella sont donc conservés chez Salmonella.

\section{Yersinia : modulation entre entrée et résistance à la phagocytose}

Dans les modèles in vitro, la première étape de l'interaction Yersinia-cellule est une adhérence intime. Le pathogène peut alors rester attaché à la 


\section{RÉFÉRENCES}

32. Hermant D, Ménard R, Arricau N, Parsot C, Popoff MY. Functional conservation of the Salmonella and Shigella effectors of entry into epithelial cells. Mol Microbiol 1995; $17: 781-9$

33. Falkow $\mathrm{S}$. Bacterial entry into eukaryotic cells. Cell 1991; 65 : 1099-102.

34. Isberg RR, Falkow S. A single genetic locus encoded by Yersinia pseudotuberculosis permits invasion of cultured animal cells by Escherichia coki K-12. Nature 1985; 317 : 262-4.

35. Isberg RR, Tran Van Nhieu G. Binding and internalization of microorganisms by integrin receptors. Trends Microbiol 1994; 2: $10-4$.

36. Hynes RO. Integrins: versatility, modulation, and signaling in cell adhesion. Cell 1992; 69: 11-25.

37. Burridge K, Turner CE, Romer LH. Tyrosine phosphorylation of paxillin and pp125 ${ }^{F A K}$ accompanies cell adhesion to extracellular matrix: a role in cytoskeletal assembly. J Cell Biol 1992; 119: 893-903.

38. Isberg RR. Discrimination between intracellular uptake and surface adhesion of bacterial pathogens. Science 1991; 252: 9348.

39. Simonet M, Richard S, Berche P. Electron microscopic evidence for in vivo extracellular localization of Yersinia pseudotuberculosis harboring the $\mathrm{pYV}$ plasmid. Infect Immun $1990 ; 58: 841-5$.

40. Guan K, Dixon JE. Protein tyrosine phosphatase activity of an essential virulence determinant in Yersinia. Science 1990; 249: 553-6.

41. Rosqvist R, Forsberg A, Wolf-Watz H. Intracellular targeting of the Yersinia YopE cytotoxin in mammalian cells induces actin microfilament disruption. Infect Immun 1991; $59: 4562-9$.

42. Michiels T, Vanooteghem JC, Lambert de Rouvoit C, China B, Gustin B, Boudry P, Cornelis GR. Analysis of virC, an operon involved in the secretion of Yop proteins by Yersinia enterocolitica. J Bacteriol 1991; 173:

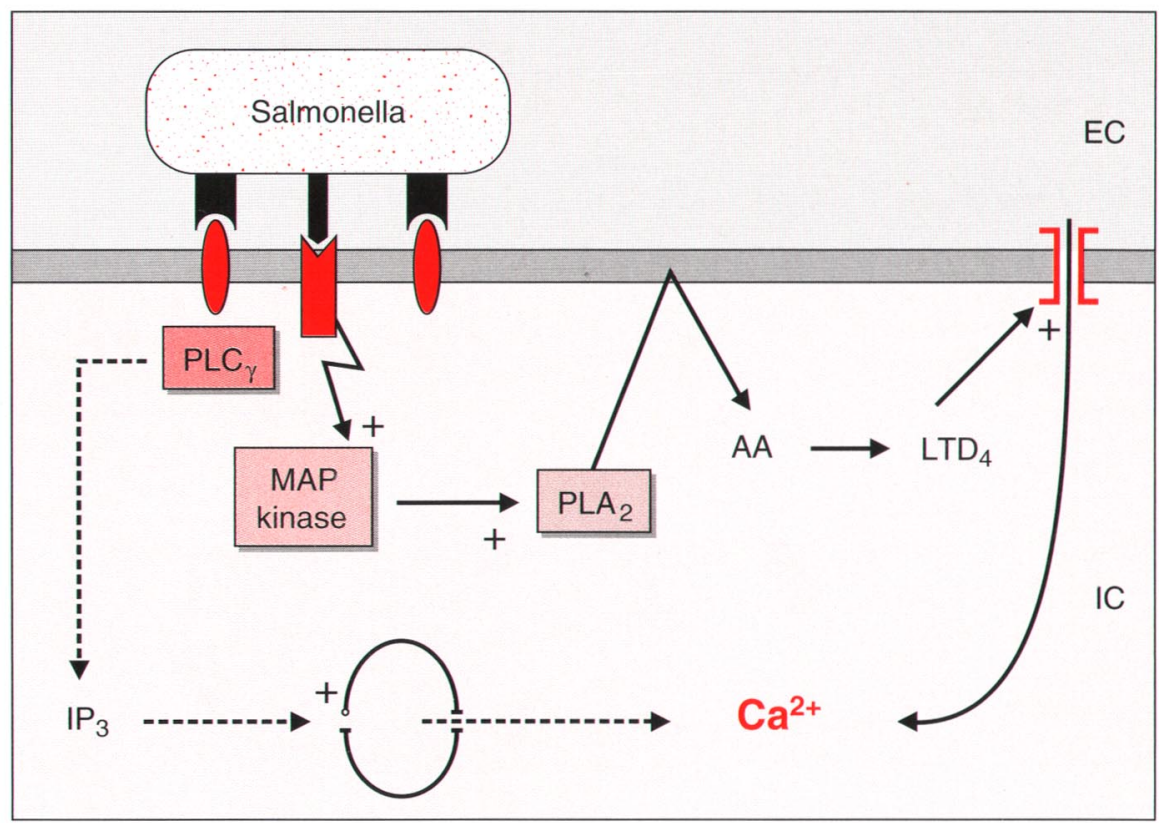

Figure 3. Modèle de signalisation induite par S. typhimurium. Quelle que soit la lignée cellulaire utilisée, une augmentation locale de la $\left[\mathrm{Ca}^{2+}\right]_{i}$ est observée après l'interaction hôte-pathogène. Les événements aboutissant à l'augmentation de la $\left[\mathrm{Ca}^{2+}\right]_{i}$ semblent dépendre de la lignée cellulaire et probablement du récepteur stimulé par la bactérie. Dans les cellules Henle-407 (traits pleins), S. typhimurium stimulerait le récepteur de I'EGF (epidermal growth factor) et entraînerait un flux entrant de $\mathrm{Ca}^{2+}$ extracellulaire comparable à celui obtenu après traitement des cellules par I'EGF et par une voie d'activation semblable. Celle-ci ferait intervenir la MAP kinase (mitogen activated protein kinase), dont les résidus tyrosine sont phosphorylés après contact bactérien, et qui activerait alors la PLA (phospholipase $A_{2}$ ). Cette activation entraînerait la production d'AA (acide arachidonique) à partir de phospholipides membranaires qui seraient convertis par la voie de la 5-lipoxygénase en un certain nombre de métabolites dont le $L T D_{4}$ (leucotriène $\left.D_{4}\right)$. Celui-ci permettrait alors un flux entrant de $\mathrm{Ca}^{2+}$ à partir de l'extérieur de la cellule. Dans les cellules HeLa (traits en pointillé), la voie de signalisation induite par S. typhimurium impliquerait la libération de $\mathrm{Ca}^{2+}$ à partir de stocks intracellulaires, induite par l'activation de la PLC $\gamma$ (phospholipase C $\gamma$ ) et la production $d^{\prime} I P_{3}$ (inositol trisphosphate). EC : milieu extracellulaire; IC: milieu intracellulaire.

surface de la cellule ou pénétrer dans la cellule. L'entrée de Yersinia, à la différence de celle de Shigella ou de Salmonella, ne nécessite pas une participation active du pathogène et n'entraîne pas la formation de projections cellulaires. Quelques minutes après le début de l'interaction, des modifications importantes de l'architecture du cytosquelette et de la forme de la cellule sont observées: progressivement, la cellule infectée s'arrondit et se détache de la matrice extracellulaire.
Trois protéines de Yersinia suffisent individuellement à permettre l'entrée dans les cellules en culture à une souche de $E$. coli de laboratoire [33]. La plus efficace est l'invasine (Inv), protéine de membrane externe se liant avec une forte affinité aux intégrines à chaîne $\beta 1$ de la cellule hôte $[34,35]$, qui lient normalement diverses protéines de la matrice extracellulaire. Une des fonctions des intégrines est de convertir les signaux extracellulaires transmis par l'adhérence d'un ligand en une 


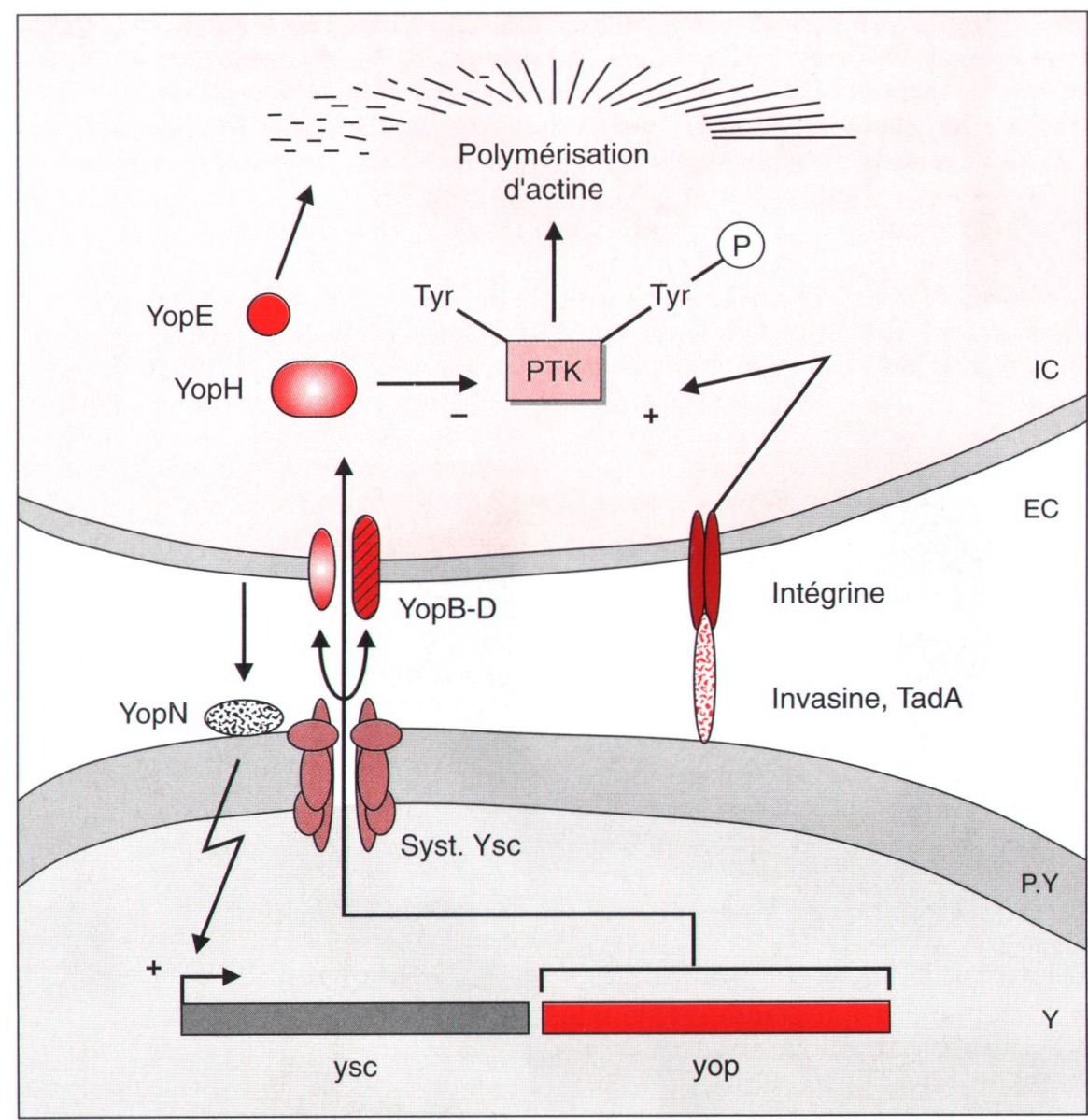

Figure 4. Modèle d'interaction Yersinia-cellule. Yersinia recouverte d'invasine s'attache aux intégrines de la surface cellulaire. La forte affinité de l'interaction invasine-intégrine permet de rompre l'association préalable intégrineligand de la matrice extracellulaire, en particulier la fibronectine. Si le nombre de récepteurs disponibles est suffisant, une interaction séquentielle survient et la bactérie est progressivement englobée grâce à la polymérisation d'actine induite via la phosphorylation de PTK (partie droite du schéma). Le contact Yersinia-cellule déclenche aussi la synthèse des protéines Yop et de leurs systèmes de sécrétion Ysc (syst. Ysc). YopN, protéine localisée à la surface des bactéries, serait le détecteur du contact avec la cellule qui met en route l'activation de la transcription des autres gènes yop et ysc ainsi que le transfert polarisé des Yop. YopB et YopD formeraient un complexe de translocation, dans le cytoplasme de la cellule, des toxines à cible intracellulaire, comme YopH et YopE. Celles-ci s'opposent à l'entrée de la bactérie, YopH en inhibant les PTK par déphosphorylation des résidus Tyr-P et YopE en dépolymérisant les filaments d'actine (partie gauche du schéma). L'infectiosité de Yersinia au cours de l'infection pourrait ainsi être modulée, en fonction de la prépondérance du système d'adhérence aux intégrines ou de celui de translocation des protéines Yop, entre attachement et entrée dans la cellule. L'invasine, produite à température ambiante, semble jouer un rôle majeur à un niveau précoce de l'infection en augmentant l'adhérence et l'efficacité d'entrée dans les cellules M [52]. A un stade plus tardif et dans les tissus, le système Yop devient prépondérant et empêche l'internalisation dans les phagocytes professionnels. EC: milieu extracellulaire; IC: milieu intracellulaire; $P Y$ : paroi bactérienne. réponse du cytosquelette [36]. Le mécanisme par lequel l'intégrine transmet le signal est inconnu, mais on connaît diverses modifications cellulaires associées à la stimulation des intégrines, comme la phosphorylation de résidus tyrosine de la PTK pp $125^{F A K}$ et de la paxilline [37], toutes deux localisées dans les plaques d'adhérence focales. La phosphorylation de résidus tyrosine de protéines cellulaires survient aussi lorsque les intégrines sont activées par leur liaison à l'invasine. Les interactions intégrine-invasine successives induiraient, via la phosphorylation de PTK, la polymérisation d'actine sous-jacente nécessaire à l'englobement progressif (en "fermeture éclair») de la bactérie [38].

En marge des nombreux travaux réalisés depuis 10 ans sur l'invasine $(\mathrm{m} / \mathrm{s}$ $n^{\circ} 10$, vol. 3, p. 626), protéine définissant le paradigme d'entrée d'une bactérie dans une cellule phagocytaire non professionnelle, les données anatomo-pathologiques se sont paradoxalement accumulées en faveur de la position majoritairement extracellulaire de Yersinia [39]. Les travaux portant sur les autres facteurs de virulence de Yersinia ont indirectement montré que l'invasine, qui permet l'entrée quand elle est exprimée seule chez $E$. coli, agit probablement en tant qu'adhésine chez les souches entéropathogènes de Yersinia. Ces souches produisent en effet une dizaine de protéines codées par un plasmide de virulence appelées Yop qui sont nécessaires à la virulence dans les modèles animaux et sont responsables des remaniements de l'architecture des cellules en culture [5]. Les protéines Yop interfèrent avec les signaux contrôlant l'architecture du cytosquelette de la cellule et induisent un signal qui s'oppose à celui induit par l'invasine (figure 4). Les mieux connues sont YopH et YopE. YopH possède une activité tyrosine-phosphatase nécessaire à la déphosphorylation rapide de résidus tyrosine phosphorylés de protéines de cellules infectées par Yersinia [40]. Le domaine C-terminal de YopH est analogue au domaine catalytique des protéines tyrosine phosphatases (PTPases) eucaryotes et il est probable que YopH s'oppose aux signaux de phagocytose en déphosphorylant les PTK activées. La protéi- 


\section{RÉFÉRENCES}

43. Bergman T, Erickson K, Galyov E, Persson C, Wolf-Watz H. The $\operatorname{lcrB}(y s c N / U)$ gene cluster of Yersinia pseudotuberculosis is involved in Yop secretion and shows high homology to the spa gene clusters of Shigella flexneri and Salmonella typhimurium. I Bacteriol 1994; 176 : 2619-26.

44. Hakansson S, Bergman T, Vanooteghem JC, Cornelis G, Wolf-Watz H. YopB and YopD constitute a novel class of Yersinia Yop proteins. Infect Immun 1993; 61 : 71-80.

45. Wattiau P, Bernier B, Deslée P, Michiels $\mathrm{T}$, Cornelis GR. Individual chaperones required for Yop secretion by Yersinia. Proc Natl Acad Sci USA 1994; 91 : 10493-7.

46. Van Gijsegem F, Genin S, Boucher C. Conservation of secretion pathways for pathogenicity determinants of plant and animal bacteria. Trends Microbiol 1993; 1 : 17580 .

47. Rosqvist R, Magnusson KE, Wolf-Watz H. Target cell contact triggers expression and polarized transfer of Yersinia YopE cytotoxin into mammalian cells. EMBO J 1994; 13: $964-72$.

48. Zychlinski A, Kenny B, Ménard R, Prévost MC, Holland IB, Sansonetti PJ. IpaB mediates macrophage apoptosis induced by Shigella flexneri. Mol Microbiol 1994; 11 : 61927.

49. Jonas D, Walev I, Berger T, Liebetrau M, Palmer M, Bhakdi S. Novel path to apoptosis: small transmembrane pores created by staphylococcal alpha-toxin in T lymphocytes evoke internucleosomal DNA degradation. Infect Immun 1994; 62: 1304-12.

50. Bliska JB, Galan JE, Falkow S. Signal transduction in the mammalian cell during bacterial attachment and entry. Cell 1993; 73: $903-20$

51. Zychlinsky A, Prévost MC, Sansonetti PJ. Shigella flexneri induces apoptosis in infected macrophages. Nature 1992; 358: 167-9.

52. Pepe JC, Miller VL. Yersinia enterocolitica invasin: a primary role in the initiation of infection. Proc Natl Acad Sci USA 1993; 90 :

ne YopE s'oppose aussi aux mécanismes de phagocytose de l'hôte. Elle est suffisante pour induire une dépolymérisation des filaments d'actine mais sa cible reste inconnue [41]. Les protéines Yop sont sécrétées dans le milieu de culture via un appareil de sécrétion appelé Ysc [5] dont la plupart des éléments sont conservés dans les sytèmes Mxi-Spa de Shigella et Inv-Spa de Salmonella [42, 43]. Pourtant, les protéines Yop purifiées incubées avec les cellules restent sans effet, suggérant qu'elles doivent être transloquées à travers la membrane de la cellule pour atteindre leur cible cellulaire. Sur la base de données structurales et d'analyse de l'action de surnageants de divers mutants yop, les protéines YopB et YopD semblent chacune nécessaire à cette fonction de translocation à travers la membrane de la cellule [44]. Ces deux produits sont codés par l'opéron $s y c D-y o p B$-yopD qui présente des similitudes avec l'opéron ipgC-ipaB-ipaC de Shigella. La protéine SycD est homologue de IpgC et se lie à chacune des protéines YopB et YopD dans le cytoplasme bactérien pour empêcher leur dégradation [45]. YopB possède deux régions hydrophobes semblables à celles de IpaB. YopD, qui s'insère probablement elle aussi dans la membrane de la cellule [44], ne présente, en revanche, aucune ressemblance avec IpaC. Cette différence explique peut-être que le système SycD-YopB/D de Yersinia ait la seule fonction de translocation de molécules effectrices et que le système IpgC-IpaB/C de Shigella comporte à la fois l'effecteur final et son appareil de translocation.

\section{Des systèmes de sécrétion proches mais adaptés à des stratégies distinctes}

Shigella, Salmonella et Yersinia sont donc dotées de systèmes de virulence très conservés et possèdent en particulier un même type d'appareil de sécrétion des effecteurs dans l'environnement, probablement en une seule étape qui court-circuite le périplasme [46]. Sur les vingt protéines de l'appareil Mxi-Spa de Shigella, 17 ont un homologue dans l'appareil Inv-Spa de Salmonella et 13 dans l'ap- pareil Ysc de Yersinia. Dans le cas de Shigella [18] et de Yersinia [47], et probablement aussi de Salmonella, l'activité du translocon est augmentée par le contact avec la cellule cible. Les bases moléculaires de la virulence sont en réalité très différentes dans les deux cas. La réponse de Shigella s'apparente à une vidange vers le milieu de protéines Ipa présynthétisées. Celle de Yersinia correspond au contraire à la mise en place de son système de virulence, comprenant l'activation de l'expression des gènes yop et le transfert polarisé de leur produit. Les Yop sont directement «injectées» dans le cytoplasme de la cellule, et non dans le milieu, probablement en raison de l'ouverture ou de l'assemblage spécifique des translocons aux seules zones d'attachement à la cellule. Bien que proches par leur structure, les translocons se sont donc adaptés aux stratégies d'infection: stockage des invasines et libération déclenchée par la cellule permettant une internalisation rapide pour Shigella, et induction de l'expression et transfert polarisé des protéines antiphagocytaires permettant une cytotoxicité au long cours pour Yersinia.

\section{Le paradoxe Shigella-Salmonella}

La conservation des effecteurs de l'entrée de Shigella et de Salmonella, les protéines Ipa et Sip, et la différence des signaux d'entrée de ces bactéries constituent aujourd'hui un paradoxe de taille. Alors que l'entrée de Shigella nécessite les petites protéines Rho mais pas d'élévation de la $\left[\mathrm{Ca}^{2+}\right]_{\mathrm{i}}$, la situation est inverse dans le cas de Salmonella. De plus, les protéines Ipa, et en particulier IpaB, sont impliquées dans l'apoptose du macrophage et dans la lyse de la membrane de la vacuole de phagocytose qui permet à Shigella de se retrouver libre dans le cytoplasme de la cellule [48], alors que Salmonella ne produit aucun de ces phénotypes. La différence de signalisation d'entrée induite par Shigella ou Salmonella pourrait être due à la spécificité différente d'un effecteur final, par exemple IpaC et SipC qui, bien que proches, possèdent des régions non conservées [31]. Elle pourrait, au contraire, être due à la différence de 
concentration d'effecteurs aux fonctions identiques, formant par exemple des pores de diamètre plus ou moins grand. L'exemple de la toxine $\alpha \mathrm{du}$ staphylocoque montre qu'une telle hypothèse est envisageable [49]. Une faible concentration de cette toxine induit la formation de pores de petite taille qui entraîne l'apoptose des lymphocytes $\mathrm{T}$ par un mécanisme mettant en jeu un flux entrant de $\mathrm{Na}^{+}$et un flux sortant de $\mathrm{K}^{+}$. A plus forte concentration, le diamètre des pores augmente, ce qui permet alors un flux entrant de $\mathrm{Ca}^{2+}$ mais n'induit plus l'apoptose. Une autre possibilité est que la différence de signalisation serait due à l'action supplémentaire d'autres facteurs chez l'une ou l'autre des bactéries, agissant en modulant l'action des protéines Ipa et/ou Sip, à la façon de l'entrée de Yersinia soumise aux effets contradictoires de l'invasine et des protéines Yop.

\section{Conclusion}

Bien qu'elles entraînent des remaniements cellulaires d'une nature différente, les bactéries des genres Shigella, Salmonella et Yersinia sont dotées d'appareils de virulence fonctionnant selon des schémas très proches, utilisés par Shigella et Salmonella pour pénétrer dans les cellules épithéliales et par Yersinia pour, au contraire, s'opposer à la phagocytose par le macrophage. Il est surprenant de constater que le système d'entrée potentiellement le plus simple, représenté par une interaction ligand bactérien de surface-récepteur cellulaire et dont l'exemple type est le couple invasine-intégrine $\beta_{1}$, n'a pas été retenu par les bactéries réellement invasives comme Shigella et Salmonella.

$\mathrm{Au}$ plan physiopathologique, la translocation de la barrière intestinale semble pouvoir être réalisée par des systèmes effecteurs différents (Invasine de Yersinia, système d'invasion de Shigella et de Salmonella) qui, après adhérence des bactéries aux cellules $\mathrm{M}$, aident au passage à travers ces cellules. L'interaction avec les cellules phagocytaires, en particulier avec les macrophages des dômes folliculaires, constitue le véritable carrefour qui va déterminer le profil évolutif ultérieur de l'infection. Yersinia empêche son internalisation et survit à la surface du macrophage qu'elle utilise pour gagner les ganglions mésentériques, Salmonella pénètre et survit dans le macrophage pour infecter le parenchyme d'organes à distance, notamment le foie et la rate, et Shigella détruit le macrophage et provoque une réaction inflammatoire locale permettant une infection entérocytaire massive (figure 1).

Au plan moléculaire, il apparaît que les altérations cellulaires, et probablement aussi les maladies, causées par ces bactéries entéropathogènes sont déterminées par la combinatoire de modules de virulence conservés. Cet effet spectaculaire de l'évolution ne devrait pas manquer d'inspirer les travaux futurs sur l'interface hôte-pathogène, qui tenteront de comparer ces différents systèmes de virulence par des approches de complémentation hétérologue et de construction de molécules hybrides. Cela devrait aboutir non seulement à une meilleure compréhension des processus de virulence des bactéries entéropathogènes mais aussi à la multiplication des outils d'étude des circuits de signalisation des cellules eucaryotes

\section{Summary}

Molecular signals triggering entry of enteroinvasive bacteria into eucaryotic cells : convergences and paradoxes

Enteropathogenic bacteria belonging to the genus Shigella, Salmonella, and Yersinia share a common capacity to cross the intestinal barrier. In in vitro models using epithelial cells grown in tissue culture dish, these bacteria induce major structural modifications of cells and are all capable of diverting cytoskeletal functions to the benefit of their entry. However, despite obvious phenotypical analogies that are reflected in the numerous homologies existing among the genes involved in cellular invasion, differences in strategies also exist which are reflected in the physiopathological diversity of infections caused by these pathogens. For each of them, the state of the art in our knowledge of the molecular and cellular bases of the host-pathogen relationship is presented. The cellular signals transduced as well as their bacterial effectors are considered. 